\title{
Switch mode to control a wheelchair through EEG signals
}

\author{
F. Velasco-Álvarez, A. Fernández -Rodríguez and R. Ron-Angevin
}

\begin{abstract}
This paper presents a switch strategy to control the movement of a Brain-Controlled Wheelchair. After the activation of a Motor Imagery task, an advance movement is started and kept until the same task stop it. This way, users with an unstable control of their EEG could be aided in handling the wheelchair.
\end{abstract}

\section{INTRODUCTION}

$\mathrm{P}$ EOPLE experiencing from numerous neurological diseases can present great disabilities. In some serious cases of disabilities, such as patients suffering Amyotrophic Lateral Sclerosis (ALS), a total loss of control of the muscles responsible of the voluntary body movements, including eye movement and breathing itself, may exist. People suffering from such deficiencies lose any possibility of communication with the external world, being the only possible alternative to give the brain a non-muscular channel that enables those people to send messages and orders to the external world. Such a system is known as a BrainComputer Interface (BCI) [1].

A BCI is based on the analysis of the brain activity, such as electroencephalographic (EEG) signals, recorded during certain mental activities, in order to control an external device. Such systems can be used for many different applications such as writing through a speller matrix [2] or driving a wheelchair. The development of a brain-controlled wheelchair (BCW) that can be handled by such patients would grant them autonomy to move through a controlled environment.

Although there are some BCWs controlled by potential P300 or SSVEP (see [3] for different examples), most systems make use of endogenous signals and especially of sensorimotor rhythm (SMR) which are usually based on discrimination of different mental tasks [3]. In these BCI systems, the number of navigation commands to control the wheelchair is associated to the number of classes to discriminate. Many studies have reported that an increasing number of classes resulted in a decrease of the classification accuracy [4]. These studies suggest that the highest classification accuracy is achieved by classifying only two classes.

In order to provide different navigation commands

This work was partially supported by the University of Málaga, by the Spanish Ministry of Economy and Competitiveness through the projects LICOM (DPI2015-67064-R (MINECO/FEDER)) and INCADI (TEC 201126395), and by the European Regional Development Fund (ERDF).

F. Velasco-Álvarez, A. Fernández-Rodríguez and R. Ron-Angevin, are with UMA-BCI group of the Dpto. Tecnología Electrónica of the University of Málaga, Málaga, Spain (fvelasco, afernandezguez, rron@uma.es) without making the BCI performance worse, our group (UMA-BCI) has been working on a paradigm based on the discrimination of only two mental tasks which allows subjects to control the selection of four different navigation commands which are sequentially presented one after the other. This paradigm has been used successfully to control a wheelchair using discrete movements [5] however, other strategies should be considered as for example, providing continuous movements [6]. In the present study, a new strategy based on switch mode control which have already been tested in a virtual wheelchair [7] is presented.

\section{MATERIAL AND METHODS}

\section{A. Participant and data acquisition}

In this preliminary study, one subject participated in this experiment (male, aged 35). EEG signals were recorded from nine active scalp electrodes placed at F3/F4, T7/T8, $\mathrm{C} 3 / \mathrm{C} 4, \mathrm{P} 3 / \mathrm{P} 4$ and $\mathrm{Cz}$, according to the 10/20 international system. The ground electrode was placed at AFz. These EEG channels were combined to produce two Laplacian channels around C3 and C4. Signals were amplified and digitized at $200 \mathrm{~Hz}$ by an actiCHamp amplifier (Brain Products GmbH, Munich, Germany).

\section{B. Initial training and signal processing}

The subject initially participated in one initial training session for calibration purposes in which, immersed in a virtual environment (VE), he had to control the displacement of a car to the right through right hand motor imagery (MI) task, or to maintain the car in a straight line through a relaxed state [5], [6]. Signal processing consisted of extracting EEG parameters and classifying them according to the two mental tasks. Feature extraction consisted of estimating the average band power of each channel in predefined frequency bands. Classification was based on a linear discriminant analysis (LDA). As a result of an LDA classification, a positive/negative value of " $\mathrm{D}$ " was computed online every $31.25 \mathrm{~ms}$ and translated into a right displacement of the car if it is positive, indicating that the trial was classified as a MI trial, or maintaining the car in a straight line if it is negative, indicating that it was a relax state trial. The same online processing was used to control the navigation paradigm.

\section{Navigation paradigm}

The navigation paradigm was identical to the one used in [5], [6]. In order to control the wheelchair, a graphical interface was presented to the users in order to offer them several navigation commands, which were selected through 
the discrimination of only two mental states. The navigation consists of a circle divided into four parts, which correspond to the possible navigation commands (move forward, F, turn right, R, move back, B and turn left, L), with a bar placed in the centre of the circle that is continuously rotating clockwise (see [5] and [6] for more details). If the classifier determines that the mental task is right-hand MI, the bar extends; otherwise (relaxed state), the bar length remains at its minimum size. When the length exceeds a selection threshold, the command pointed by the bar is selected. Due to the main objective being to control a real wheelchair, the graphical interfaces used were replaced by an audio-cued interface, allowing to select a command without a graphical interface.

Two different configuration commands can be select with this interface. In continuous mode, once the command is selected, the wheelchair moves in a continuous way (see [6]). In switch mode, once a command is selected, the movement starts. The main difference is that when the bar is shortened under the selection threshold, the movement does not stop, but it is kept until the user enlarges the bar length above the selection threshold again (carrying out a MI mental task); at that moment, the movement stop.

In some situations, continuous mode could have some disadvantages. If the user wants to move forward during a long period in order to cover medium or long distances, he/she needs to keep the effort of the MI task in order to keep the wheelchair moving forward. The aim of this preliminary study is to check the usefulness of this brainswitch mode.

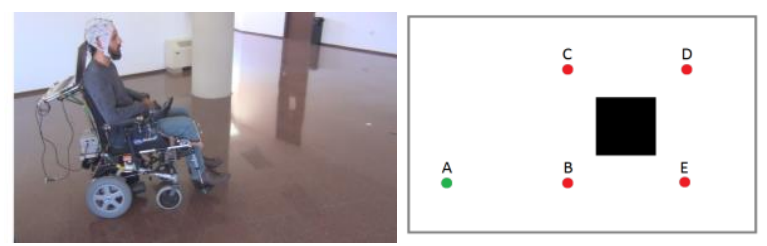

Fig. 1. The subject driving the wheelchair (left) and the demarcated path to follow (right).

\section{Experimental procedure}

Prior to driving the robotic wheelchair, the subject went through a training schedule: a first session for calibration purpose and a second training session in a VE. This second session consisted of freely navigating in the VE in order to get used to the control interface. First, the subject navigated the virtual wheelchair using the audiovisual interface and in a second task using the audio-cued interface only.

After the training sessions, the subject participated in one experimental session using a switch mode to control the advances of the robotic wheelchair (Fig. 1, left), while the turn and backward commands were executed in a discrete way $\left(90^{\circ}\right.$ turns and $1 \mathrm{~m}$ backward motion). Using small squares drawn on the floor, we demarcated a path consisting of four $3 \mathrm{~m}$ stretches, one $6 \mathrm{~m}$ stretch and four turns (Fig. 1, right).

\section{RESULTS AND DISCUSSION}

The obtained results of the experimental session are shown in Table I. For each stretch (comprising the turn after the advance) three columns are showed: i) the time spent, ii) the selected commands and iii) the precision, calculated as the ratio between the correct selections and the total selections. Considering the way the interface managed the advance commands, one selection of the F command implied two activations of the MI task.

The subject needed 13 commands while the path could optimally be completed with 9 commands. He made 2 mistakes that he amended with 2 extra commands. In stretches B-C and C-D the subject needed two advances (instead of only one). As the table shows, the high precision value indicates that the paradigm allowed the subject to choose the commands he wanted without difficulty.

This same subject participated in a previous experiment in continuous mode, not showing any control.

TABLE I

NAVIGATION RESULTS

\begin{tabular}{cccc}
\multicolumn{4}{c}{ NAVIGATION RESUlTs } \\
\hline \hline Stretch & Time (s) & Commands & Precision \\
\hline A-B & 131 & F, R (error), L, L & 0.75 \\
B-C & 51 & F, F (error), R & 0.66 \\
C-D & 119 & F, F, R & 1 \\
D-E & 62 & F, R & 1 \\
E-A & 64 & F & 1 \\
\hline Total & 427 & 13 commands & 0.84 \\
\hline \hline
\end{tabular}

\section{CONCLUSION}

Even when this is a preliminary result, the switch mode seems to be an appropriate way to make easier the forward movement of the wheelchair compared to the continuous mode.

\section{REFERENCES}

[1] J. Wolpaw, N. Birbaumer, D. J. McFarland, G. Pfurtscheller, and T. M. Vaughan, "Brain Computer Interfaces for communication and control," Clin. Neurophysiol., vol. 113, pp. 767-791, 2002.

[2] L. A. Farwell and E. Donchin, "Talking off the top of your head: Toward a mental prosthesis utilizing event-related brain potentials," Elec.. Clin. Neurophysiol., vol. 70, no. 6, pp. 510-523, 1988.

[3] L. Bi, X.-A. Fan, and Y. Liu, "EEG-Based Brain-Controlled Mobile Robots: A Survey," IEEE Trans. Human-Machine Syst., vol. 43, no. 2, pp. 161-176, 2013.

[4] B. Obermaier, C. Neuper, C. Guger and G. Pfurtscheller. (2001, Information transfer rate in a five-classes brain-computer interface. IEEE Trans Neural Systems and Rehab Eng. 9(3), pp. 283-288.

[5] S. Varona-Moya et.al., Wheelchair Navigation with an Audio-cued, Two-Class Motor Imagery-based Brain-Computer Interface System, proceedings of the 7th International IEEE EMBS Conf on Neural Engineering, 2015 (NER).

[6] R. Ron-Angevin, A. Fernández-Rodríguez and F. Velasco-Álvarez, "Brain-controlled wheelchair through discrimination of two mental tasks". SAI Intelligent Systems Conference 2016, to be published

[7] Velasco-Álvarez, F., Ron-Angevin, R., Da Silva-Sauer, L., \& SanchaRos, S. (2010). Brain-computer interface: Comparison of two paradigms to freely navigate in a virtual environment through one mental task. In Proceedings of the IB2COM. 